\title{
Discovery of non-radial pulsations in the spectroscopic binary Herbig Ae star RS Chamaeleontis ${ }^{\star}$
}

\author{
T. Böhm ${ }^{1}$, W. Zima ${ }^{2}$, C. Catala ${ }^{3}$, E. Alecian ${ }^{4}$, K. Pollard ${ }^{5}$, and D. Wright ${ }^{5}$ \\ ${ }^{1}$ Laboratoire Astrophysique de Toulouse-Tarbes, Université de Toulouse, CNRS, 14 avenue Edouard Belin, 31400 Toulouse, France \\ e-mail: boehm@obs-mip.fr \\ 2 Instituut voor Sterrenkunde, KU Leuven, Celestijnenlaan 200D, 3001 Leuven, Belgium \\ 3 LESIA, Observatoire de Paris-Meudon, 5 place Jules Janssen, 92195 Meudon, France \\ 4 Dept. of Physics, Royal Military College of Canada, PO Box 17000, Stn Forces, Kingston, K7KK 7B4 Canada \\ 5 Dept. of Physics and Astronomy, University of Canterbury, Private Bag 4800, Christchurch, New Zealand
}

Received 2 December 2008 / Accepted 15 January 2009

\begin{abstract}
Context. To understand the origin of stellar activity in pre-main-sequence Herbig Ae/Be stars and to get a deeper insight into the interior of these enigmatic stars, the pulsational instability strip of Palla and Marconi is investigated. In this article we present a first discovery of non radial pulsations in the Herbig Ae spectroscopic binary star RS Cha.

Aims. The goal of the present work is to detect non-radial pulsations in a Herbig Ae star for the first time directly by spectrographic means and to identify the largest amplitude pulsation modes.

Methods. The spectroscopic binary Herbig Ae star RS Cha was monitored in quasi-continuous observations during 14 observing nights (Jan. 2006) at the $1 \mathrm{~m}$ Mt. John (New Zealand) telescope with the Hercules high-resolution echelle spectrograph. The cumulative exposure time on the star was $44 \mathrm{~h}$, corresponding to 255 individual high-resolution echelle spectra with $R=45000$. Least-square deconvolved spectra (LSD) were obtained for each spectrum, representing the effective photospheric absorption profile modified by pulsations. Difference spectra were calculated by subtracting rotationally broadened artificial profiles, these residual spectra were analysed and non-radial pulsations detected. A subsequent analysis with two complementary methods, namely Fourier Parameter Fit (FPF) and Fourier 2D (F2D) has been performed and first constraints on the pulsation modes derived.

Results. For the very first time, we discovered by direct observational means using high-resolution echelle spectroscopy, non-radial oscillations in a Herbig Ae star. In fact, both components of the spectroscopic binary are Herbig Ae stars and both show NRPs. The FPF method identified 2 modes for the primary component with (degree $\ell$, azimuthal order $m$ ) couples ordered by decreasing probability: $f_{1}=21.11 \mathrm{~d}^{-1}$ with $(\ell, m)=(11,11),(11,9)$ or $(10,6)$ and $f_{2}=30.38 \mathrm{~d}^{-1}$ with $(\ell, m)=(10,6)$ or $(9,5)$. The F2D analysis indicates for $f_{1}$ a degree $\ell=8-10$. For the secondary component, the FPF method identified 3 modes with $(\ell, m)$ ordered by decreasing probability: $f_{1}=12.81 \mathrm{~d}^{-1}$ with $(\ell, m)=(2,1)$ or $(2,2), f_{2 b}=19.11 \mathrm{~d}^{-1}$ with $(\ell, m)=(13,5)$ or $(10,5)$ and $f_{3}=24.56 \mathrm{~d}^{-1}$ with $(\ell, m)=(6,3)$ or $(6,5)$. The F2D analysis indicates for $f_{1}$ a degree $\ell=2$ or 3 , but proposes a contradictory identification of $f_{2 b}$ as a radial pulsation $(\ell=0)$.
\end{abstract}

Key words. stars: pre-main-sequence - stars: oscillations - stars: individual: RS Cha - stars: binaries: spectroscopic

\section{Introduction}

Asteroseismology represents a modern tool for studying the stellar interiors of the enigmatic intermediate mass $\left(\sim 2-8 M_{\odot}\right)$ pre-main-sequence (PMS) stars, so-called Herbig Ae/Be stars (Herbig 1960; Strom et al. 1972; Finkenzeller \& Mundt 1984; Finkenzeller \& Jankovics 1984). Since their first systematic classification in 1960 (Herbig 1960), this group of young stars has been extensively studied, but one of the major questions remains unanswered: how can the intense stellar activity, the strong stellar winds (Praderie et al. 1982; Catala et al. 1986a; Catala \& Kunasz 1987; Böhm \& Catala 1995; Böhm et al. 1996), and the many highly variable emission lines observed in the spectra of these stars be explained?

Magnetism is frequently invoked as being responsible for active stellar phenomena. The position of the Herbig stars in the HR diagram indicates that they are in the radiative phase of their contraction towards the main-sequence (Iben 1965;

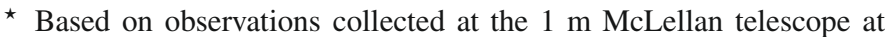
Mt John, NZ.
Gilliland 1986), and should in principle not possess any outer convective zone. In its absence, the classical magnetic dynamo mechanism could not be responsible for the observed active phenomena. Moreover, recent spectropolarimetric observations of Herbig stars indicate the presence of significant large-scale magnetic fields only in a small fraction of them (Wade et al. 2005; Catala et al. 2007; Alecian et al. 2008). This fraction, close to $10 \%$, is consistent with a magnetic field distribution similar to that of the main-sequence stars. In that scenario, magnetic Herbig Ae stars would be the progenitors of main-sequence $\mathrm{Ap} / \mathrm{Bp}$ stars. These recent results agree with the primordial fossil field hypothesis. Unless magnetic fields in Herbig Ae/Be stars are of very complex nature and can therefore not be detected by spectropolarimetric means, in opposition to clearly organised dipolar fields, the pure magnetic origin of activity in these stars is more than doubtful.

For many years non-classically generated magnetic fields have been used as a potential explanation for the observed active phenomena. Even if the presence of complex fields can not be ruled out it is of major importance to investigate other 
possible external or internal origins of this tremendous amount of dissipated energy, as witnessed by chromospheres and coronae, but also variable spectral lines, winds and bipolar jets.

The only way of studying in detail the internal stellar structure is the analysis and the modelling of stellar pulsations, if observed. As an example, PMS stars gain their energy from gravitational contraction and therefore differ significantly from post-main-sequence stars having already processed nuclear material - stellar pulsations are sensitive to these differences of internal stellar structure (see e.g. Suran et al. 2001). As of today, the internal stellar structure of PMS stars is not yet well constrained. Since few years the existence of pulsating intermediate mass PMS stars is known (e.g. Breger 1972; Kurtz \& Marang 1995; Donati et al. 1997). This observational result motivated Marconi \& Palla (1998) to investigate the pulsation characteristics of HR 5999 theoretically, which enabled them to predict the existence of a pre-main-sequence instability strip, which is being crossed by most of the intermediate mass PMS objects for a significant fraction of their evolution to the main-sequence. This strip covers approximately the same area in the HR diagram as the $\delta$ Scuti variables. Zwintz (2008) compared, based on photometry, the observational instability regions for pulsating pre-main-sequence and classical $\delta$ Scuti stars and concluded that the hot and cool boundaries of both HR diagram instability regions seem to coincide. This preliminary result deserves further study by aim of full asteroseismological approach based on spectroscopy.

As of today, more than 30 intermediate-mass PMS stars have revealed to be pulsating at time-scales typical of $\delta$ Scuti stars (see e.g. Kurtz \& Marang 1995; Kurtz \& Catala 2001; Donati et al. 1997; Böhm et al. 2004; Marconi et al. 2002; Ripepi \& Marconi 2003; Zwintz \& Weiss 2003; Catala 2003, and references therein).

RS Chamaeleontis is a bright spectroscopic eclipsing binary star. Both components are Herbig Ae PMS stars of similar mass (close to $1.9 M_{\odot}$ ). Recently the age of RS Cha has been determined to $6_{-1}^{+2}$ Myr (Luhman \& Steeghs 2004), which verifies it's PMS nature. Andersen (1975) already reported small amplitude radial velocity variations on top of the binary radial velocity curve for both components of RS Cha, suggesting the possible presence of stellar pulsations. Photometric observations by McInally \& Austin (1977) revealed short-term variations in at least one of the two components, possibly linked to stellar pulsations. Very recently, Alecian et al. (2005) reported radial velocity variations in the residual velocity frame (cleaned for orbital velocity) with amplitudes up to a few $\mathrm{km} \mathrm{s}^{-1}$ and periods of the order of $1 \mathrm{~h}$, indicative of $\delta$ Scuti type pulsations.

The aim of our study of the two components of RS Cha is to provide a first set of asteroseismic constraints for forthcoming non-radial pulsation models by determining unambiguously a higher number of periodicities and identifying, in a second step, the corresponding pulsation modes with their respective degree $\ell$ and azimuthal number $m$.

To achieve this goal, we decided to perform high resolution spectroscopic observations on a large time basis and with optimised time coverage.

Section 2 reviews previous related work, Sect. 3 describes the observations and data reduction, Sect. 4 summarises results of the orbit determination, Sect. 5 reveals the detection of nonradial pulsations in both components of RS Cha, Sects. 6 and 7 present frequency analysis and mode identification in the primary and secondary component, respectively. A discussion is proposed and a conclusion is drawn in Sect. 8.
Table 1. Parameters of RS Cha.

\begin{tabular}{lccc}
\hline \hline Parameter & Primary & Secondary & References $^{a}$ \\
\hline$M / M_{\odot}$ & $1.89 \pm 0.01$ & $1.87 \pm 0.01$ & {$[1]$} \\
$R / R_{\odot}$ & $2.15 \pm 0.06$ & $1.87 \pm 0.01$ & {$[1]$} \\
$T_{\text {eff }}[\mathrm{K}]$ & $7638 \pm 76$ & $7228 \pm 72$ & {$[2]$} \\
$\log \left(L / L_{\odot}\right)$ & $1.15 \pm 0.09$ & $1.13 \pm 0.09$ & $L=4 \pi R^{2} \sigma T^{4}$ \\
$\log (g)\left[\mathrm{cm} \mathrm{s}^{-2}\right]$ & $4.05 \pm 0.06$ & $3.96 \pm 0.06$ & $g=M \mathrm{G} / R^{2}$ \\
$v \sin i\left[\mathrm{~km} \mathrm{~s}^{-1}\right]$ & $64 \pm 6$ & $70 \pm 6$ & {$[1]$} \\
$P_{\text {orb }}[\mathrm{d}]$ & \multicolumn{2}{c}{1.67} & {$[1]$} \\
$i[\mathrm{deg}]$ & \multicolumn{2}{c}{$83.4 \pm 0.3$} & {$[3]$} \\
{$[\mathrm{Fe} / \mathrm{H}]$} & \multicolumn{2}{c}{$0.17 \pm 0.01$} & {$[1]$} \\
\hline
\end{tabular}

a References: [1] Alecian et al. (2005); [2] Ribas et al. (2000); [3] Clausen \& Nordstrom (1980).

Table 2. Log of the observations at Mt John Observatory, NZ, in Jan. 2006.

\begin{tabular}{ccccc}
\hline \hline $\begin{array}{c}\text { Date } \\
(1)\end{array}$ & $\begin{array}{c}\mathrm{JD}_{\text {first }} \\
(2)\end{array}$ & $\begin{array}{c}\mathrm{JD}_{\text {last }} \\
(3)\end{array}$ & $\begin{array}{c}N_{\text {spec }} \\
(4)\end{array}$ & $\begin{array}{c}S / N_{\text {Range }} \\
(5)\end{array}$ \\
\hline Jan. 09 & 3745.0101 & & 1 & 120 \\
Jan. 10 & 3745.9544 & 3745.9733 & 2 & $80-100$ \\
Jan. 12 & 3747.9537 & 3748.1779 & 28 & $70-90$ \\
Jan. 13 & 3749.0013 & 3749.1930 & 23 & $100-120$ \\
Jan. 14 & 3749.8911 & 3749.9911 & 12 & $70-90$ \\
Jan. 15 & 3750.8921 & 3751.1919 & 31 & 150 \\
Jan. 16 & 3751.8958 & 3752.1970 & 29 & $90-120$ \\
Jan. 19 & 3754.9015 & 3755.2006 & 33 & $100-130$ \\
Jan. 20 & 3755.9245 & 3756.1993 & 25 & $90-120$ \\
Jan. 21 & 3756.9068 & 3757.0386 & 14 & $60-150$ \\
Jan. 22 & 3757.9004 & 3758.1621 & 32 & $120-170$ \\
\hline
\end{tabular}

(1) Date of the observation; (2) Julian date (mean observation, $2450000+)$ of the first and (3) last stellar spectrum of the night, respectively; (4) Number of spectra; (5) typical range of $S / N\left(\right.$ pixel $^{-1}$ ) at $550 \mathrm{~nm}$.

\section{Previous related work}

The pre-main-sequence spectroscopic eclipsing binary RS Cha has been studied extensively throughout the last years. Thanks to its eclipsing nature and the known inclination angle the system has fully been calibrated Alecian et al. (2005, 2007a,b). Table 1 summarises the main results.

\section{Observations}

The analysis presented in this paper is based on a 14 night observing run in January 2006 at the $1 \mathrm{~m} \mathrm{Mt} \mathrm{John} \mathrm{telescope}$ equipped with the Hercules echelle spectrograph. We obtained quasi-continuous single-site observations of the target star during these 2 weeks and obtained a total of 255 individual stellar echelle spectra, each spectrum having an individual exposure time of $10 \mathrm{~min}$. The star was observed with high resolution spectroscopy at $R \approx 45000$ and covering the wavelength area from 457 to $704 \mathrm{~nm}$, spread over 44 orders. The detector was a $1 \mathrm{k} \times 1 \mathrm{k}$ Site CCD. The highest $S / N\left(\right.$ pixel $^{-1}$ ) values we obtained reached 210 on Jan 16th, corresponding to almost 300 per resolved element (2 pixels); typical values of $S / N$ (pixel $^{-1}$ ) ranged around 80-150. Table 2 summarises the log of the observations.

The general observing strategy was to obtain as many $10 \mathrm{~min}$ observations of the target star during the night as possible. Before the beginning and after the end of the night we obtained complete sets of calibrations: in general 6 bias images, 6 tungsten light flat field spectra, and, as close as possible to the first 
stellar spectrum, 2 ThAr arc spectra (the calibration set at the end of the night being scheduled in the opposite order).

In order to guarantee the precision of the wavelength solution we decided to regularly interleave ThAr arc spectra during the night (every two hrs).

Most of the data reduction was carried out following standard reduction procedures using the "ESPRIT" spectroscopic data reduction package (Donati et al. 1997). This package also makes use of the "optimal extraction algorithm" (Horne 1986). Heliocentric velocity and Julian date correction was performed. The intrinsic wavelength calibration accuracy achieved with the "ESPRIT" 2D-polynomial fit procedure is better than $0.22 \mathrm{pm}$ (standard deviation, i.e. $2.2 \mathrm{~m} \AA$, corresponding to $120 \mathrm{~ms}^{-1}$ at $5500 \AA$ ) for the Hercules data set.

The best way to correct the $2 \mathrm{D}$-wavelength polynomial for evolution during the night due to the intrinsic spectral instability of the instrument was revealed to be the computation of least square deconvolved LSD profiles (Donati et al. 1997) of the more than 100 telluric water vapour lines contained in each stellar spectrum and to cross correlate them with respect to the first spectrum of the night. The precision of the above correction is around $50 \mathrm{~ms}^{-1}$ (see Appendix A in Böhm \& Hirth 1997). All spectra were then corrected with their individual timedependent correction to the initial wavelength calibration based on the ThAr arc lamp spectrum acquired at the beginning of each night. More details about this procedure can be read in Böhm et al. (2004).

The next step of the data reduction was to calculate for all 255 stellar spectra photospheric LSD-profiles, using a mask corresponding to the spectral type $\mathrm{A} 7$, but taking care to eliminate from the line list all transitions showing non-photospheric behaviour (Hydrogen Balmer lines, He I D3, many FeII lines, $\mathrm{NaI} \mathrm{D}, \ldots)$. The equivalent photospheric profiles contain eventually multiplex information of 1930 individual photospheric lines, most of them being very weak lines. Typically, a spectrum of RS Cha with $S / N=150\left(\right.$ pixel $\left.^{-1}\right)$ at $5500 \AA$ yields a typical $S / N$ value of 1400 per velocity step (or pixel) in the resulting LSD profile. The accuracy of the radial velocity determination on an individual LSD profile scales, to the first order, with the inverse of the square root of the weighted multiplex gain and is therefore estimated to be as low as $12 \mathrm{~ms}^{-1}$; however, the limiting factor in the precision of the overall radial velocity correction is clearly the spectra-to-spectra cross correlation of telluric line LSD profiles. We therefore estimate the finally achieved precision in radial velocity to be around $50 \mathrm{~ms}^{-1}$ for each individual stellar LSD profile of this data set, which is largely enough for our spectroscopic study.

Figure 1 shows a typical LSD profile of RS Cha with both primary and secondary component well separated in radial velocity.

\section{Orbit determination with circularised binary approximation}

The binary LSD profile was then fitted with a double rotational profile (see e.g. Gray 2005) with an IDL procedure. This yielded the precise heliocentric radial velocities of the two components for all cases except the fully merged profiles. In a second step, the velocities were then fitted with a circularised orbit (i.e. sinusoidal radial velocity curves) which can be seen in Fig. 2. Orbital parameters are listed in Table 3. The less massive secondary component was identified based on its slightly larger orbital amplitude.

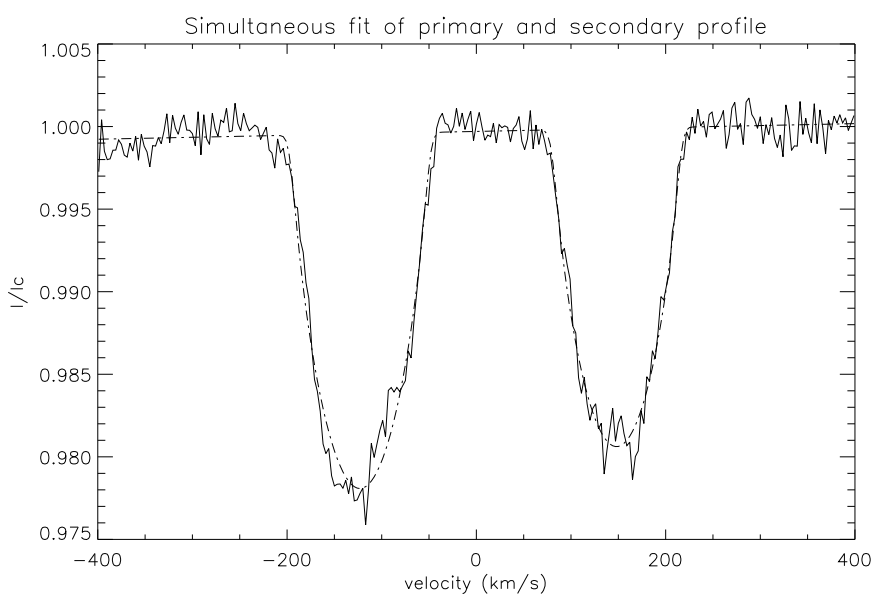

Fig. 1. Binary spectrum from Jan. 12th $2006(\Delta$ HJD $=3745.9575)$ (thick line) superimposed with a simultaneous rotational fit of both components. The little bumps in the core of the line profiles are indicative of non-radial pulsations.

It was interesting to determine the orbital period and its phase shift with respect to the work published previously. Based on the method described in Alecian et al. (2005) we calculated the observed minus computed $(\mathrm{O}-\mathrm{C})$ timings of the first conjunction and added our new point to Fig. 3 showing it's evolution over a time span of 30 years. We can observe that the curve seems to decline slightly in gradient, indicative of either the presence of a third body in the RSCha system or of the signature of changes in the orbital period of the order of $\Delta P / P=(7.5 \pm 0.7) \times 10^{-6}$. Further observations in the next years would be required to better characterise these orbital variations and to understand their origin.

\section{Detection of non-radial pulsations in both components}

Finally, we extracted the two binary components of each LSD profile along its individual orbital radial velocity in order to obtain two files with centred dynamical spectra revealing only the intrinsic stellar profile variations. Figures 4 and 5 reveal the deviations from the mean (rotational) profile for three different nights. It can be clearly seen that bumps move through the line profile in a quite complex manner. Figure 4 (primary) reveals a more diagonal trend of the bump movement (bottom-left to upright) than Fig. 5 (secondary). As will be later shown, these features are due to several high-degree non-radial pulsation modes. This represents a first direct detection of non-radial pulsations in Herbig Ae stars by spectroscopic means.

The colour coding in Figs. 4 and 5 represents a dynamical range of about $0.5 \%$ of the continuum. The dashed vertical lines at the respective $\pm v \sin i$ indicate roughly the border of profiles enlarged by rotation. The date of the first reference spectra of each night can be seen in Table 2 .

It must be noted at this stage that low frequency remnants of imprecise orbital fitting subsist which can not easily be accounted for with a circularised binary orbit fit, revealing perhaps the presence of an additional yet undetected multiple component. 


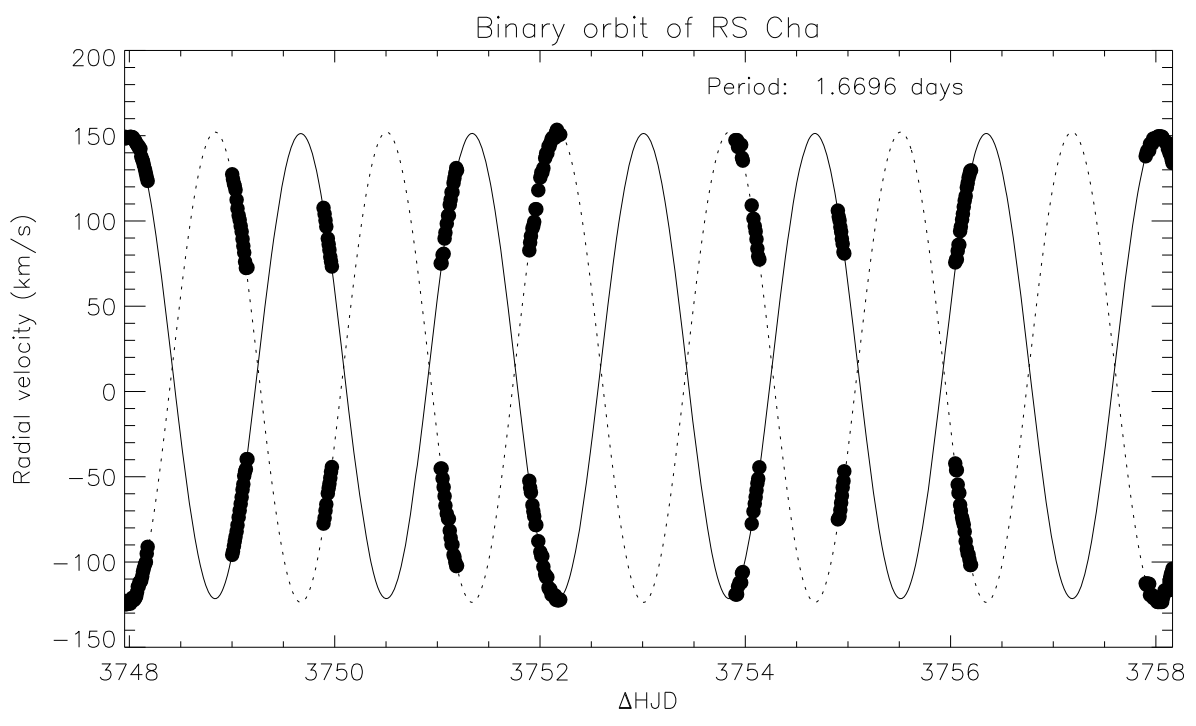

Fig. 2. Orbital movement of the spectroscopic binary components of RS Cha. $\triangle \mathrm{HJD}=$ HJD - 2450000.0 . The continuous and dotted lines show the adjusted circular orbits of the primary and secondary component, respectively. The dots represent the observed radial velocity values of both components. Dot sizes do not represent error bars and have been selected only for better visibility of the figure. Typical error bars are of the order of $50 \mathrm{~ms}^{-1}$ and are too small to be represented in this plot.
Table 3. Orbital parameters of both components of RS Cha.

\begin{tabular}{lcc}
\hline \hline Parameter & Primary component & Secondary component \\
\hline$\Delta \mathrm{HJD}^{a}$ periastron & 3750.5044 & 3749.6695 \\
Period (days) & $1.66965 \pm 0.00003$ & identical \\
Amplitude $\left(\mathrm{km} \mathrm{s}^{-1}\right)$ & 136.4159 & 137.9077 \\
\hline
\end{tabular}

${ }^{a} \Delta \mathrm{HJD}=\mathrm{HJD}-2450000.0$.

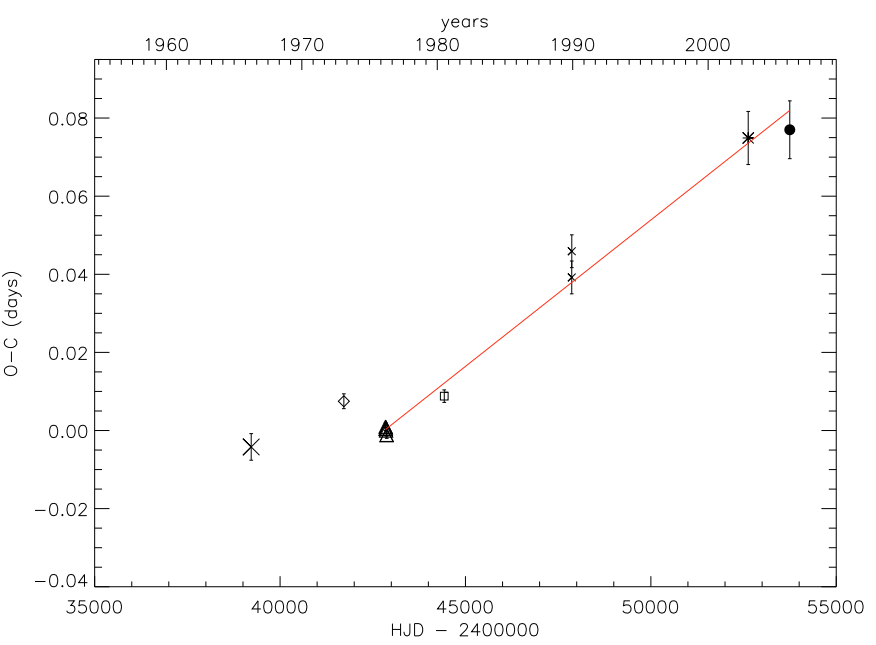

Fig. 3. Observed minus computed $(\mathrm{O}-\mathrm{C})$ timings of the first conjunction over 30 years. One full circled point corresponding to the new 2006 data set was added to the figure taken from Alecian et al. (2005).

\section{Pulsational analysis of the primary component}

At this stage, the spectra have been corrected for the radial velocity variations caused by the binary orbit of RS Cha. We selected only spectra for the frequency analysis and mode identification that do not show any blending of the LSD profiles of the two components, i.e., the situation when the difference in orbital velocities was larger than approximately the sum of the two projected rotational velocities, $\Delta v \geq v_{1} \sin i+v_{2} \sin i$. The useful Doppler velocity range for the analysis was thus between -55 and $+55 \mathrm{~km} \mathrm{~s}^{-1}$ which excluded the continuum. A set of 161 spectra covering $35 \mathrm{~h}$ was finally selected for the analysis. See Table 4 for a log of the observations.

\subsection{Frequency analysis of the primary component}

We have used the software package FAMIAS (Zima 2008) for the search for periodicities in the line profile and for the identification of the pulsation modes using the Fourier parameter fit (FPF) method (Zima 2006). The search for periodicities was carried out by using Discrete Fourier Transformation (DFT) and multiperiodic non-linear least-squares fitting (LSF), a method which fits a sum of sinusoids to the variations. Both methods assume sinusoidal variations, which in general is a good approximation for pulsation modes having low amplitudes.

In order to search for pulsation frequencies we applied the following iterative procedure: in the Fourier spectrum, the frequency peak having highest amplitude is selected and the data pre-whitened with a least-squares fit that included this peak and all previously found frequencies. If several peaks have similar amplitudes, e.g., due to aliasing, the peak which results in the lowest residuals is selected. The search is stopped if none of the remaining peaks reaches a $S / N$ of above 4 (Breger et al. 1993), corresponding to a $99.9 \%$ confidence level.

Since the dispersion range that we selected for analysis did not extend towards the continuum, we were not able to properly compute line moments. We therefore computed the centre of gravity $(\mathrm{CoG})$ of the line profile, which is numerically similar to the first moment, to study its temporal variations. Furthermore, we examined the pixel-intensity-variations (PIV) across the line profile for the frequency search. We studied the PIV by computing the mean Fourier spectrum across the line profile. The significance of a frequency peak was determined by computing its $S / N$ in the wavelength bin where it has the largest amplitude. The two methodologies are complementary in the way that the $\mathrm{CoG}$ is more sensitive to low-degree pulsation modes $(\ell \leq 4)$, whereas the PIV are also sensitive to modes of higher degree.

The Fourier spectrum of the CoG time series showed many peaks in the low-frequency domain between 0 and $6 \mathrm{~d}^{-1}$ (see Fig. 6). These peaks are associated to nightly zero point velocity shifts of the line profile and are likely caused by the uncertainty of the binary orbit fitting. No formally significant peaks were found above $6 \mathrm{~d}^{-1}$.

The frequency analysis of the PIV also revealed several peaks in the low frequency domain (see Fig. 7). Here, they are clearly associated with the uncertainties of the orbit fitting. We found significant frequencies at 0.599 and at $1.197 \mathrm{~d}^{-1}$, which corresponds to the orbital frequency and twice thereof. After 

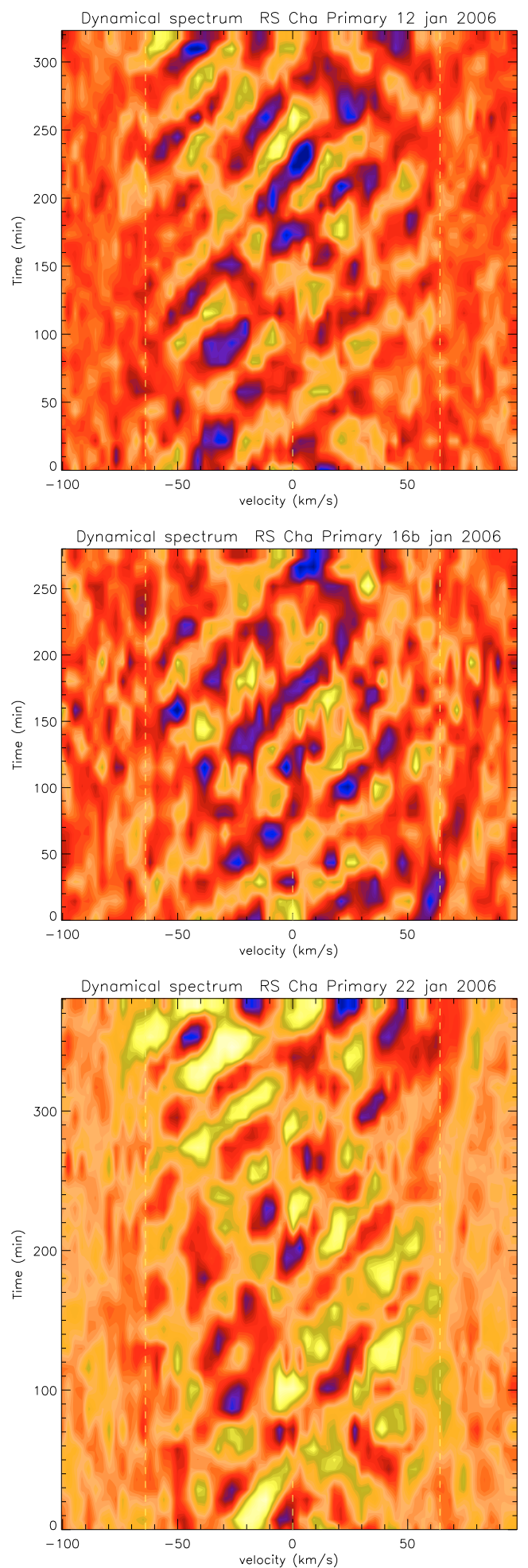

Fig. 4. Line profile variations due to the oscillations for the primary component of RS Cha. The deviations from the mean intensity are displayed for three different nights (Jan. 12th, 16th and 22nd 2006).

pre-whitening with these terms, two other significant frequencies could be detected in the data: $f_{1}=21.11$ and $f_{2}=30.38 \mathrm{~d}^{-1}$. After further pre-whitening, no additional frequencies having a $S / N$ above 4 could be found. Table 5 lists the frequencies detected in the primary component. The value $A_{\text {PIV }}$ was the integral of the pixel-amplitude across the line profile between -55
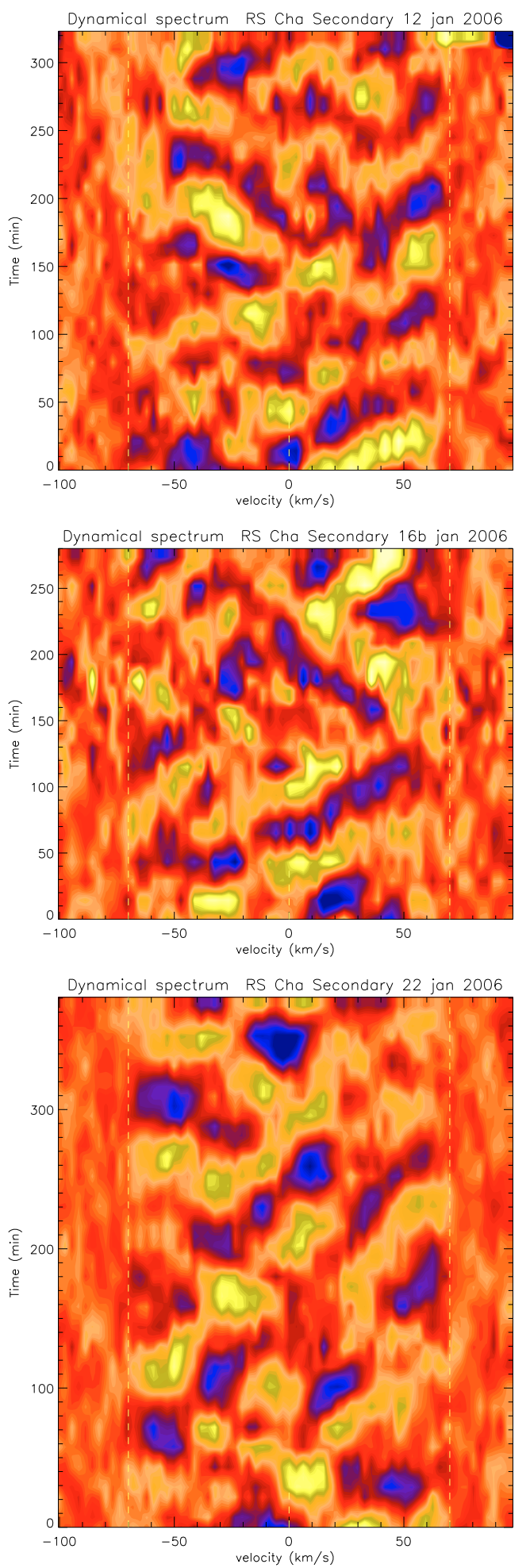

Fig. 5. Same as Fig. 4 for the secondary component of RS Cha.

and $55 \mathrm{~km} \mathrm{~s}^{-1}$, and for each frequency the $S / N$ was computed for the wavelength bin where its amplitude was highest.

\subsection{Mode identification with the FPF method}

We carried out a mode identification of the two detected frequencies with the FPF method. This method makes use of the fact that stellar oscillations cause intensity variations in a rotationally broadened line profile due to the Doppler effect. It is capable to 
Table 4. Data subset used for the frequency analysis.

\begin{tabular}{ccccc}
\hline \hline $\begin{array}{c}\text { Date } \\
(1)\end{array}$ & $\begin{array}{c}\mathrm{JD}_{\text {first }} \\
(2)\end{array}$ & $\begin{array}{c}\mathrm{JD}_{\text {last }} \\
(3)\end{array}$ & $\begin{array}{c}\mathrm{N}_{\text {spec }} \\
(4)\end{array}$ & $\begin{array}{c}\text { Duration } \\
(5)\end{array}$ \\
\hline Jan. 12 & 3747.9537 & 3748.1607 & 26 & 4.97 \\
Jan. 13 & 3749.0013 & 3749.1099 & 13 & 2.60 \\
Jan. 14 & 3749.8911 & 3749.9411 & 6 & 1.20 \\
Jan. 15 & 3751.0417 & 3751.1838 & 16 & 3.41 \\
Jan. 16 & 3751.8958 & 3752.1861 & 28 & 6.97 \\
Jan. 18 & 3753.9037 & 3754.1257 & 16 & 5.33 \\
Jan. 19 & 3754.9015 & 3754.9259 & 8 & 1.32 \\
Jan. 20 & 3756.0609 & 3756.1912 & 16 & 3.13 \\
Jan. 22 & 3757.9004 & 3758.1621 & 32 & 6.28 \\
\hline Total & & & 161 & 35.2 \\
\hline
\end{tabular}

(1) Date (2006); (2) Julian date (2450 000+) of the first; and (3) last observation of the night; (4) number of spectra; and (5) summed exposure time (hrs) of the night.

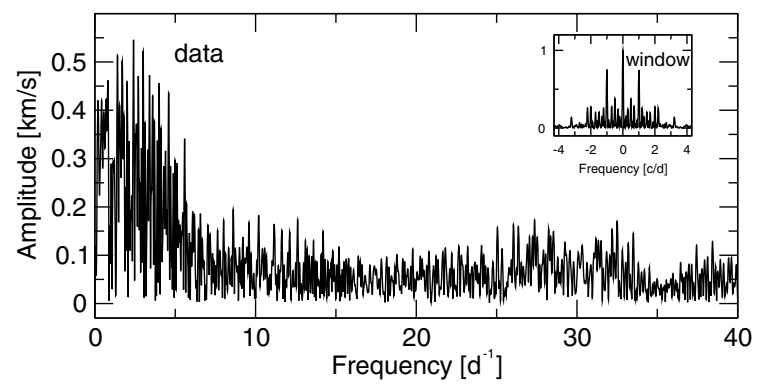

Fig. 6. Amplitude spectrum of the CoG time-series of the primary component. No significant frequency peaks could be detected in these data.

determine the spherical degree, $\ell$, and the azimuthal order, $m$, of a pulsation mode from a time series of line profile variations. The contribution of each pulsation frequency to the observed line profile variations can be de-composed into its Fourier parameters, the zero-point, amplitude, phase across the line profile. These Fourier parameters are determined from a multi-periodic least-squares fit across the line profile, i.e., for each wavelength bin, and have a characteristic distribution according to the related $\ell$ and $m$-values. By comparing the observed Fourier parameters and their counterparts computed from synthetic line profile variations, we can determine the pulsational characteristics of a pulsation mode. The reduced chi-square values $\chi_{v}^{2}$ of the different solutions are computed from the complex amplitudes and their uncertainties across the line profile. For details we refer to Zima (2006).

The synthetic line profiles were computed assuming that the pulsations can be described by a superposition of spherical harmonics. Furthermore, we assume a Gaussian intrinsic line profile, one infinitesimal thin line forming region, and a symmetry axis of the pulsation field that coincides with the rotation axis.

We searched the following parameter space [min; max; step]: $\ell \in[0 ; 15 ; 1], m \in[-\ell ; \ell ; 1]$ for each $\ell$, pulsation velocity amplitude $a \in[0 ; 60 ; 0.5] \mathrm{km} \mathrm{s}^{-1}, v \sin i \in[30 ; 80 ; 1]$, and width of Gaussian intrinsic profile $\sigma \in[1 ; 15 ; 1] \mathrm{km} \mathrm{s}^{-1}$. The inclination value of the RS Cha system is well known (Clausen \& Nordstrom 1980). Assuming that the inclination of the rotation axis of the components is identical to that of the system, we set it as fixed parameter to $i=83.4^{\circ}$ during the search for the optimum in the parameter space.

Our analysis with the FPF method revealed that both detected frequencies are high-degree pro-grade pulsation modes. Due to the uncertainties of the derived amplitude and phase across the line profile, we were not able to unambiguously

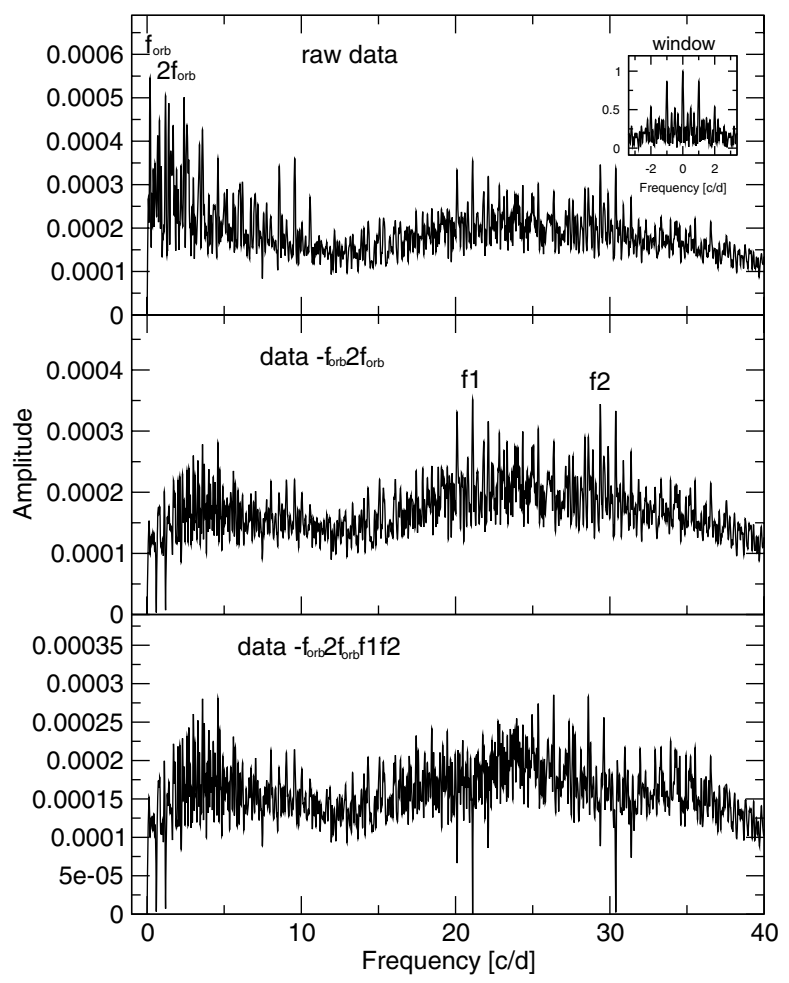

Fig. 7. Amplitude spectra of the pixel-by-pixel intensity time-series of the primary component. The different panels show the data after subsequent pre-whitening of the indicated frequency peaks.

Table 5. Frequencies detected in the pixel-by-pixel intensity variations of the primary component of RS Cha.

\begin{tabular}{lccc}
\hline \hline ID & $\begin{array}{c}\text { Freq. } \\
\mathrm{d}^{-1}\end{array}$ & $\begin{array}{c}A_{\text {PIV }}^{a} \\
\mathrm{~km} \mathrm{~s}^{-1}\end{array}$ & $S / N$ \\
\hline$f_{\text {orb }}$ & 0.599 & 0.106 & 13.9 \\
$2 f_{\text {orb }}$ & 1.197 & 0.040 & 4.5 \\
$f_{1}$ & 21.11 & 0.040 & 4.5 \\
$f_{2}$ & 30.38 & 0.038 & 4.0 \\
\hline
\end{tabular}

${ }^{a} A_{\text {PIV }}$ represents the integral of the pixel-amplitude across the line profile.

identify $\ell$ and $m$ but are left with a number of solutions, which still will constrain possible theoretical seismic models. The five best solutions of each frequency are listed in Table 6 . Figure 8 shows the Fourier parameters of the observed and best fitting model to the two detected frequencies. The fact that the model does not take into account all parameters of the atmosphere implies that a perfect fit to the observations cannot be achieved. For example, we did not include equivalent width variations of the intrinsic line profile due to the significant increase in the free parameter space and the associated determination uncertainty. As can be seen for $f_{2}$ in Fig. 8 (or later on for $f_{3}$ in Fig. 13), a phase jump of close to $2 \pi$ occurs in the right wing of the profile fit, due to the uncertainty of the determination and the poor amplitude fit in this area of the profile.

We identified both frequencies as pro-grade modes of high degree. The best solution for $f_{1}$ identifies it as sectoral mode of $\ell=m=11$, whereas the best identification for $f_{2}$ is a tesseral mode with $\ell=10$ and $m=6$. The uncertainties of this identification are larger for $f_{2}$ than for $f_{1}$ and in the range of \pm 1 for both quantum numbers. 

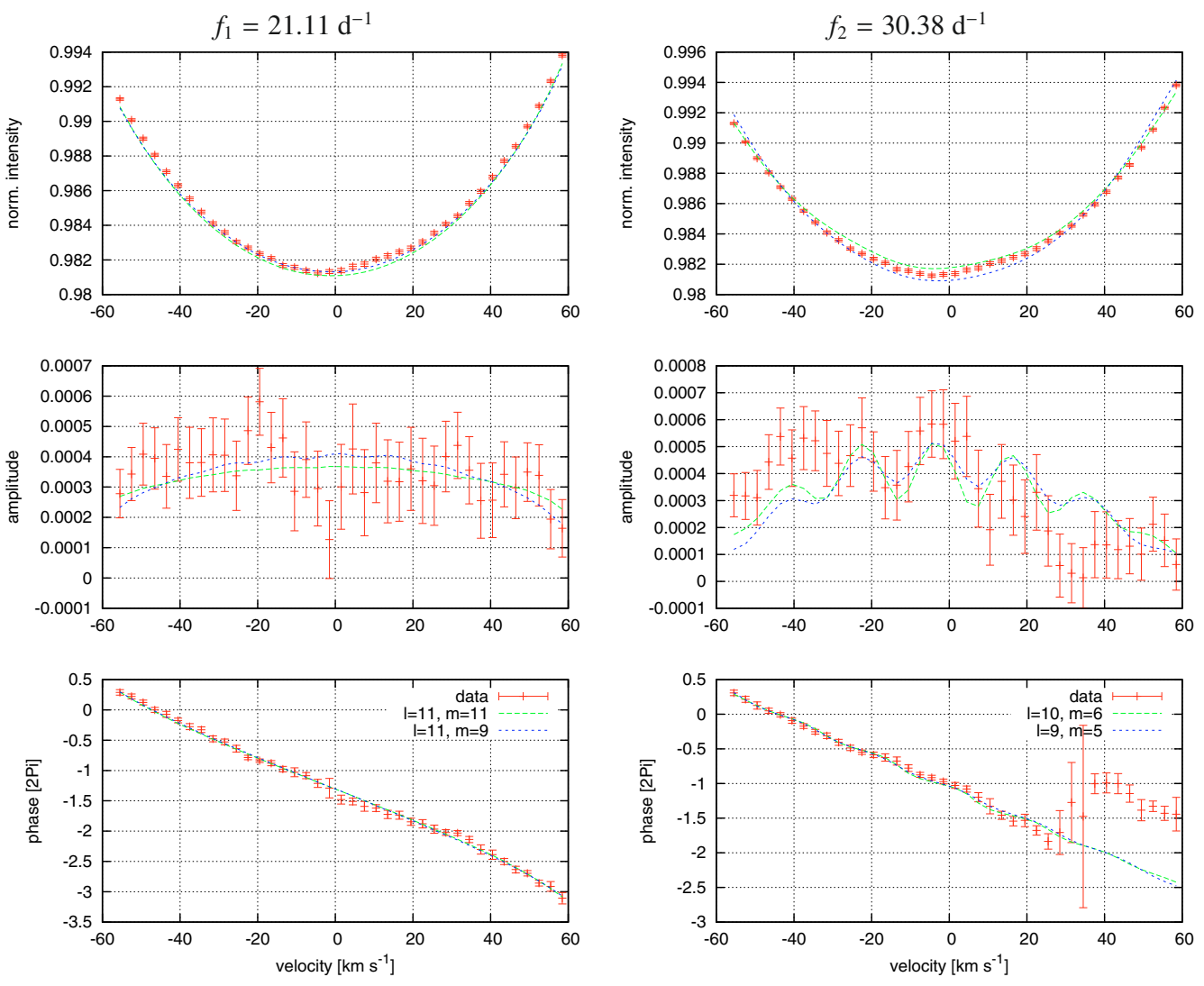

Fig. 8. Fourier parameters across the line profile of the pulsation frequencies detected in the primary component of RS Cha. Each panel shows from top to bottom: zero-point, amplitude and phase. For both frequencies, the two best fits to the observed zero point, amplitude in units of the continuum and the phase distribution in units of $2 \pi$ are shown. The uncertainties are the formal errors computed from the least-squares fitting.

Table 6. Mode parameters derived for the primary component of RS Cha from the application of the FPF method.

\begin{tabular}{cccccc}
\hline \hline$\chi_{v}^{2}$ & $\ell$ & $m$ & $a$ & $v \sin i$ & $\sigma$ \\
\hline \multicolumn{5}{c}{$f_{1}=21.11 \mathrm{~d}^{-1}$} \\
\hline 0.70 & 11 & 11 & 0.5 & 68 & 3 \\
0.74 & 11 & 9 & 1.5 & 68 & 4 \\
0.79 & 10 & 6 & 4.0 & 67 & 3 \\
0.85 & 11 & 7 & 4.5 & 71 & 5 \\
1.04 & 10 & 8 & 1.5 & 66 & 5 \\
\hline \multicolumn{6}{c}{$f_{2}=30.38 \mathrm{~d}^{-1}$} \\
\hline 1.21 & 10 & 6 & 52.0 & 67 & 12 \\
1.33 & 9 & 5 & 20.0 & 66 & 11 \\
1.37 & 11 & 7 & 50.0 & 71 & 12 \\
1.41 & 8 & 4 & 9.5 & 66 & 7 \\
1.48 & 11 & 9 & 43.0 & 70 & 13 \\
\hline
\end{tabular}

For each pulsation mode, the five best solutions are shown.

We derived a projected rotational velocity of $v \sin i=68 \pm$ $2 \mathrm{~km} \mathrm{~s}^{-1}$ which agrees quite well with the previously determined value by Alecian et al. (2005) and implies a rotation frequency of $0.63 \pm 0.01 \mathrm{~d}^{-1}$.

\subsection{Mode identification with the Fourier 2D method}

A complementary, and very direct method of analysing the non radial pulsation modes present in the LSD-spectra time series consists of applying the Fourier 2D method (Kennelly 1994; Kennelly et al. 1996), resulting in a representation of the data in the frequency - apparent $m$ space. The apparent $m$ is related to the structure of the modes present at the stellar surface, without being identical to the usual azimuthal orde $m$. The original work by Kennelly (1994) showed in fact that apparent $|m|$ scales as $\ell+2$ for values close to zero, as $\ell+1$ for values lower than 10 and as $\ell$ for values above 10 . Basically, this method consists of doing subsequently a spectral and a temporal Fourier analysis. All LSD profiles of the time series were normalised to the same equivalent width, in order to eliminate the effects of large scale variations not related to the signature of pulsations. The time average of the mean profiles was then constructed and subtracted from each mean profile, yielding a time series of normalised residual profiles. Before doing the 2 dimensional Fourier analysis, each residual profile was interpolated on a grid representing stellar longitudes, transforming velocities across the line profile into longitudes on the stellar equator using the relation $\Delta v=v \sin i \sin \phi$, where $\Delta v$ is the velocity position within the LSD profile with respect to the rest wavelength of the star and $\phi$ the stellar longitude angle of the star in spherical coordinates (under assumption that $\sin i$ is close to unity, which is the case for RS Cha). The Fourier transform being very sensitive to gaps in the temporal sampling, we selected only continuous series of spectra within a night, and interpolated them on a $2^{n}$ regularly spaced temporal grid, where $\mathrm{n}$ is selected in order to avoid overor under-sampling. As a result, we obtained F2D spectra for each suitable night. In order to take into account the individual 

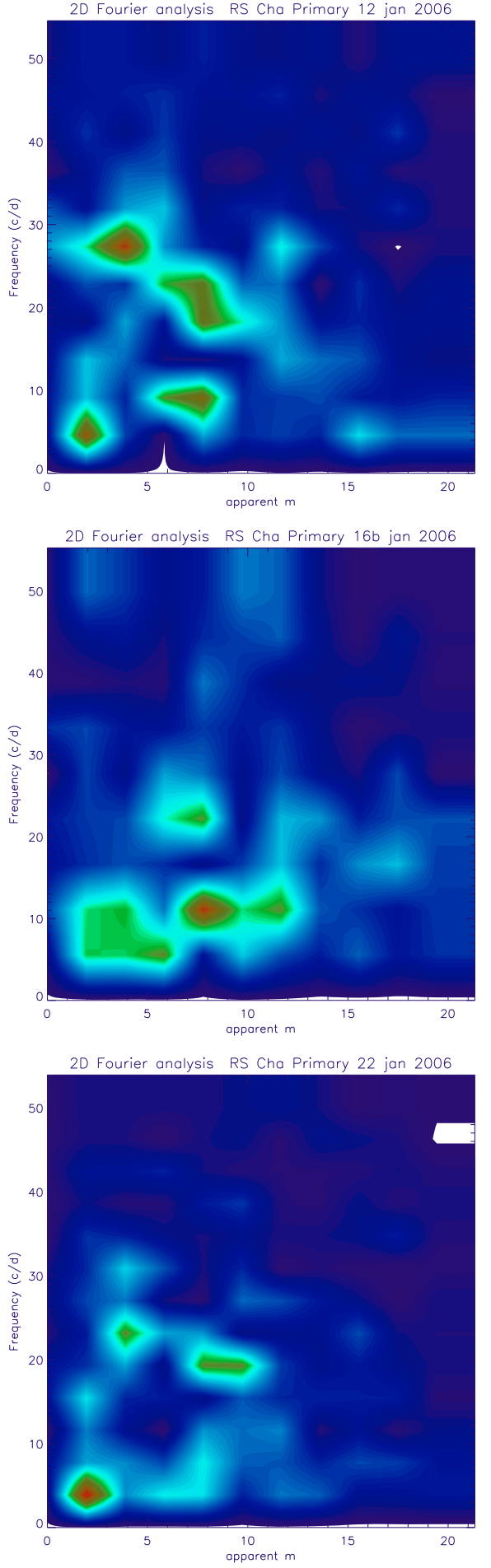

Fig. 9. F2D analysis on the primary component of RS Cha for the nights of Jan. 12th, 16th and 22nd 2006. Apparent $|\mathrm{m}|$ scales as $\ell+2$ for values close to zero, as $\ell+1$ for values lower than 10 and as $\ell$ for higher values (Kennelly 1994). White patches are only due to the applied cutoff values of the representation and have no further meaning.

quality of each night, we decided to combine the spectra of the three nights with a time-basis of more than 250 continuously observed minutes in the following way:

$F 2 D_{\text {tot }}=\sqrt{\sum_{j=1}^{n} a_{j}\left|F 2 D_{j}\right|^{2}}$

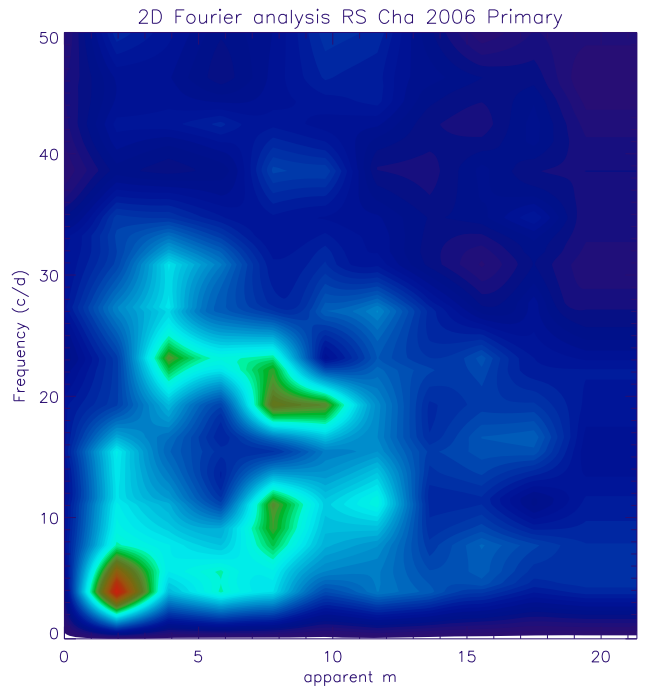

Fig. 10. Weighted F2D periodogram of the primary component of RS Cha for the nights of Jan. 12th, 16th and 22nd 2006.

where $a_{j}$ is a normalised weighting coefficient:

$a_{j}=\frac{n_{\text {spec }_{j}} \overline{S / N}_{j}^{2}}{\sum_{k=1}^{n} n_{\text {spec }_{k}} \overline{S / N}_{k}^{2}}$

and $\overline{S / N}_{j}$ the average signal to noise ratio per spectrum for a given night, $n_{\text {spec }}$ the number of spectra per night and $n$ the number of exploitable nights (in the sense of the Fourier 2D analysis). The coefficient $a_{j}$ reflects therefore the total cumulated stellar photon flux per night, normalised to the cumulated photon flux of the full observing run. While this expression is not mathematically rigorous it reflects to some extent the quality of the individual nights.

Figure 9 shows the nightly F2D analysis for the nights of 12th, 16th and 22nd of January 2006, representing the modulus of the complex Fourier 2D amplitude in the frequency $\left(\mathrm{d}^{-1}\right)-$ apparent $|m|$ space. Kurtz \& Müller (1999) estimate the uncertainty in the frequency to be approximately $1 /(4 \Delta T)$, where $\Delta T$ is the time span of the data set. In our case for a typical observing night of 300 min continuous observations, this would yield an uncertainty of $1.2 \mathrm{~d}^{-1}$ in the frequency determination. This, together with large-scale beating effects on the pulsational signature and significant $S / N$ ratio variations from one night to another might account for slightly different positions of frequencies in the F2D space. However, the "general" pattern is preserved from night to night, as can be seen in Fig. 9, and the weighted F2D periodogram in Fig. 10 exhibits the strongest recurring oscillation modes.

Table 7 summarises the results for the primary component based on the F2D analysis.

The F2D method confirms the existence of the high degree mode $f_{1}$ determined by the FPF method $\left(f_{1}=21.11 \mathrm{~d}^{-1}\right)$ and indicates a degree $\ell$ of $8-10$, within the error bars of the FPF determination. While single night F2D periodograms show some high frequency signal around 29. $\mathrm{d}^{-1}$ (e.g. night of Jan. 12th), which could correspond to $f_{2}$ (FPF), this is not confirmed systematically. A low frequency signal around $4 . \mathrm{d}^{-1}$ is very likely a fundamental oscillation frequency due to the window function ( $t_{\text {obs }}$ typically $1 / 4 \mathrm{~d}$ ) and is present in all F2D periodograms seen in Fig. 9. Other frequencies found in the weighted F2D 
Table 7. Frequency search and mode identification. Summary of the weighted F2D analysis on the primary component.

\begin{tabular}{cccc}
\hline \hline $\begin{array}{c}\mathrm{d}^{-1} \\
(1)\end{array}$ & $\begin{array}{c}\text { FPF frequency } \\
(2)\end{array}$ & $\begin{array}{c}\text { App. }|m| \\
(3)\end{array}$ & $\begin{array}{c}\ell \\
(4)\end{array}$ \\
\hline 20. & $f_{1}$ & $8-10$ & $8-10$ \\
24. & & 4 & 2 or 3 \\
12. & & 8 & 8 \\
4. & & 2 & 0 \\
\hline
\end{tabular}

(1) Frequency $\left( \pm 1.2 \mathrm{~d}^{-1}\right)$; (2) corresponding FPF frequency; (3) apparent $|m|$; (4) expected $\ell$ value.

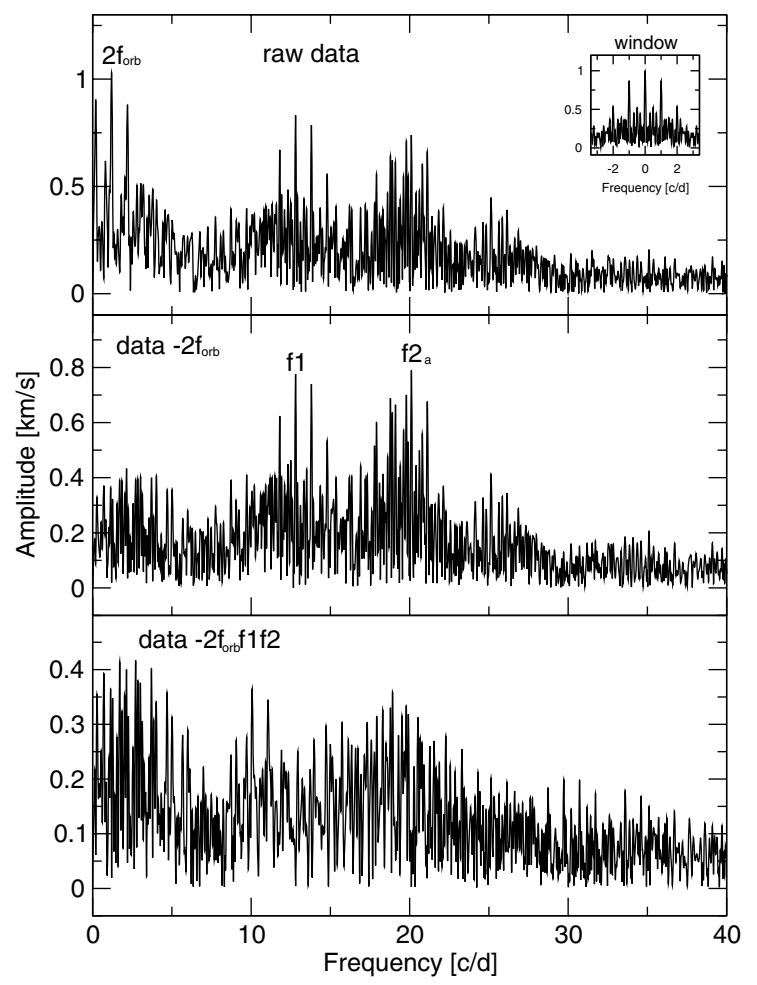

Fig. 11. Amplitude spectrum of the CoG time-series of the secondary component. The different panels show the data after subsequent prewhitening of the indicated frequency peaks.

periodogram around $12 . \mathrm{d}^{-1}$ and $24 . \mathrm{d}^{-1}$ (with different apparent $|m|$ values) have not been revealed by the FPF method.

\section{Pulsational analysis of the secondary component}

The analysis of the secondary component was performed on a selected data subset, following the same selection rules as for the primary component. This procedure left 165 spectra that were suited for the further analysis. We only used the central parts of the line profile between $\pm 70 \mathrm{~km} \mathrm{~s}^{-1}$ in Doppler velocity, the extreme wings bearing not much useful information for the mode identification. We again used the CoG of the profile to estimate the radial velocity variations. We carried out the frequency search and mode identification in the same manner as for the primary component.

\subsection{Frequency analysis of the secondary component}

The Fourier spectrum of the CoG time series (see Fig. 11) shows a dominant frequency peak with an amplitude of $1 \mathrm{~km} \mathrm{~s}^{-1}$ at twice the orbital frequency $1.19 \mathrm{~d}^{-1}$. After pre-whitening of this

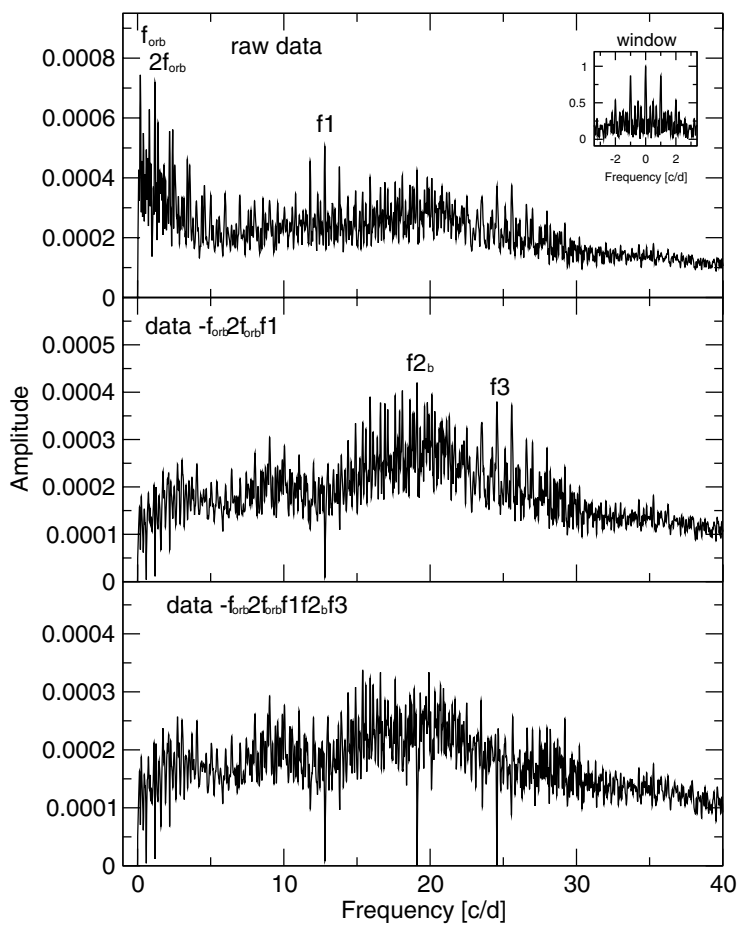

Fig. 12. Amplitude spectra of the pixel-by-pixel intensity time-series of the secondary component. The different panels show the data after subsequent pre-whitening of the indicated frequency peaks.

Table 8. Frequencies detected in the pixel-by-pixel intensity variations of the secondary component of RS Cha.

\begin{tabular}{lccc}
\hline \hline ID & $\begin{array}{c}\text { Freq. } \\
\mathrm{d}^{-1}\end{array}$ & $\begin{array}{c}A_{\mathrm{PIV}}^{a} \\
\mathrm{~km} \mathrm{~s}^{-1}\end{array}$ & $S / N$ \\
\hline$f_{\text {orb }}$ & 0.599 & 0.060 & 5.7 \\
$2 f_{\text {orb }}$ & 1.197 & 0.138 & 8.2 \\
$f_{1}$ & 12.81 & 0.066 & $3.9^{1}$ \\
$f_{2 b}$ & 19.11 & 0.061 & 5.7 \\
$f_{3}$ & 24.56 & 0.054 & 4.0 \\
\hline
\end{tabular}

${ }^{a} A_{\mathrm{PIV}}$ represents the integral of the pixel-amplitude across the line profile.

${ }^{1}$ This frequency has a $S / N=5$ in the CoG time-series. Therefore, we relaxed the significance criterion for this frequency in the PIV.

frequency, the low frequency domain below $6 \mathrm{~d}^{-1}$ does not show any more significant frequencies and has a much lower noise level than the primary component. Strong $1 \mathrm{~d}^{-1}$-aliasing complicates the detection of further frequencies. The highest remaining peak is at $12.8 \mathrm{~d}^{-1}$ with an amplitude of $0.78 \mathrm{~km} \mathrm{~s}^{-1}$, but the oneday aliasing peak at $13.9 \mathrm{~d}^{-1}$ has a similar amplitude. A similar situation occurs at $20.1 \mathrm{~d}^{-1}$. We selected two peaks that had a $S / N$ above 4 and that resulted in the lowest residuals after prewhitening, $f_{1}=12.81$ and $f_{2 a}=20.11 \mathrm{~d}^{-1}$. After pre-whitening with these frequencies, no further significant peaks can be found in the Fourier spectrum.

The analysis of the PIV across the line profile (see Fig. 12) revealed a dominant peak at $0.19 \mathrm{~d}^{-1}$, which is a one-day aliasing of twice the orbital frequency at $1.19 \mathrm{~d}^{-1}$. Also the orbital frequency had a significant amplitude in the PIV which, as has been mentioned before, is a result of imperfect orbital fitting. We pre-whitened the data with these two frequencies and found $f_{1}=12.81 \mathrm{~d}^{-1}$ as highest remaining peak. After further prewhitening, the highest peak is at $f_{2 b}=19.11 \mathrm{~d}^{-1}$ which is a one-day aliasing of $f_{2 a}$. Pre-whitening with $f_{2 b}$ resulted in much 

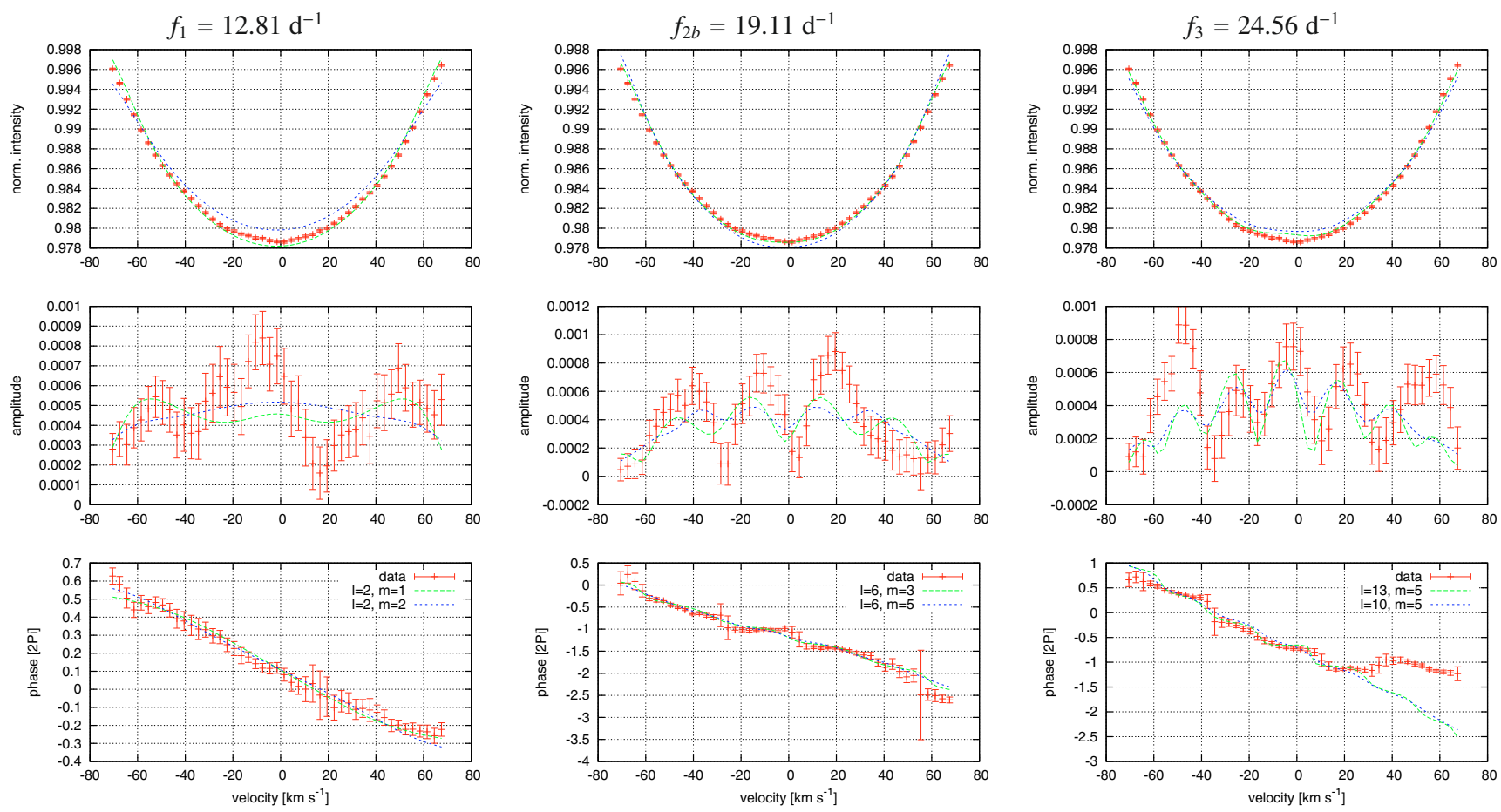

Fig. 13. Fourier parameters across the line profile of the pulsation frequencies detected in the secondary component of RS Cha. Each panel shows from top to bottom: zero-point, amplitude in units of the continuum and phase in units of $2 \pi$. For both frequencies, the two best fits are shown. The uncertainties are the formal errors computed from the least-squares fitting.

Table 9. Mode parameters derived for the secondary component from the application of the FPF method.

\begin{tabular}{cccccc}
\hline \hline$\chi_{v}^{2}$ & $\ell$ & $m$ & $a$ & $v \sin i$ & $\sigma$ \\
\hline \multicolumn{5}{c}{$f_{1}=12.81 \mathrm{~d}^{-1}$} \\
\hline 1.03 & 2 & 1 & 20.2 & 69 & 7.5 \\
1.06 & 2 & 2 & 3.7 & 74 & 15.0 \\
1.34 & 1 & 1 & 3.7 & 69 & 6.6 \\
1.35 & 3 & 2 & 18.9 & 74 & 15.0 \\
1.91 & 3 & 3 & 3.1 & 74 & 15.0 \\
\hline \multicolumn{5}{c}{$f_{2 b}=19.11 \mathrm{~d}^{-1}$} \\
\hline 4.06 & 13 & 5 & 22.6 & 72 & 7.5 \\
4.23 & 10 & 5 & 15.6 & 74 & 7.5 \\
4.30 & 9 & 4 & 12.3 & 70 & 4.7 \\
4.33 & 12 & 4 & 20.3 & 68 & 6.6 \\
4.45 & 10 & 10 & 1.0 & 71 & 7.5 \\
\hline \multicolumn{5}{c}{$f_{3}=24.56 \mathrm{~d}^{-1}$} \\
\hline 2.09 & 6 & 3 & 20.9 & 70 & 7.5 \\
2.15 & 6 & 5 & 7.7 & 69 & 5.6 \\
2.21 & 7 & 4 & 28.6 & 71 & 11.2 \\
2.34 & 7 & 6 & 13.7 & 74 & 11.2 \\
2.34 & 6 & 6 & 1.6 & 69 & 11.2 \\
\hline
\end{tabular}

For each pulsation mode, the five best solutions are shown

lower residuals than pre-whitening with $f_{2 a}$, which contradicts the frequency analysis of the CoG. Since we used the PIV for the mode identification with the FPF method, we accepted $f_{2 b}$ as the "true" frequency. After pre-whitening, one more frequency at $f_{3}=24.56 \mathrm{~d}^{-1}$ with a $S / N$ of 4 was detected. No other significant peaks could be extracted from the data. The detected frequencies are listed in Table 8 . The integral of the pixel-amplitudes were computed between -70 and $70 \mathrm{~km} \mathrm{~s}^{-1}$. For each frequency, the
Table 10. Frequency search and mode identification. Summary of the weighted F2D analysis for the secondary component.

\begin{tabular}{cccc}
\hline \hline $\mathrm{d}^{-1}$ & FPF frequency & app. $|m|$ & $\ell$ \\
$(1)$ & $(2)$ & $(3)$ & $(4)$ \\
\hline 19. & $f_{2 b}$ & 2 & 0 \\
12. & $f_{1}$ & 4 & 2 or 3 \\
\hline
\end{tabular}

(1) Frequency; (2) corresponding FPF frequency; (3) apparent $|m|$; (4) expected $\ell$ value.

Table 11. Frequency search and mode identification. Summary of the main results for the dominant frequencies of each component.

\begin{tabular}{|c|c|c|c|}
\hline \multicolumn{4}{|c|}{ Primary component } \\
\hline & $\mathrm{d}^{-1}$ & Identification FPF & Identification $^{b}$ F2D \\
\hline \multirow[t]{3}{*}{$\begin{array}{l}f_{1} \\
f_{2} \\
\end{array}$} & $\begin{array}{l}21.11 \\
30.38 \\
\end{array}$ & $\begin{array}{c}\text { best }^{a}(\ell, m) \\
(11,11),(11,9),(10,6) \\
(10,6),(9,5),(11,7)\end{array}$ & $\ell=8-10$ \\
\hline & & Secondary component & \\
\hline & $d^{-1}$ & Identification FPF & Identification $^{b}$ F2D \\
\hline $\begin{array}{l}f_{1} \\
f_{2 b} \\
f_{3}\end{array}$ & $\begin{array}{l}12.81 \\
19.11 \\
24.56\end{array}$ & $\begin{array}{c}\text { best }^{a}(\ell, m) \\
(2,1),(2,2) \\
(13,5),(10,5) \\
(6,3),(6,5)\end{array}$ & $\begin{array}{c}\ell=2 \text { or } 3 \\
\ell=0\end{array}$ \\
\hline
\end{tabular}

${ }^{a}$ The $(\ell, m)$ couples indicated for the FPF method correspond to the best identification, in decreasing order.

${ }^{b}$ For each of the frequencies detected by the FPF method, we identified an $\ell$ value as detected by the F2D method.

$S / N$ was computed for the wavelength bin where the amplitude was highest. 

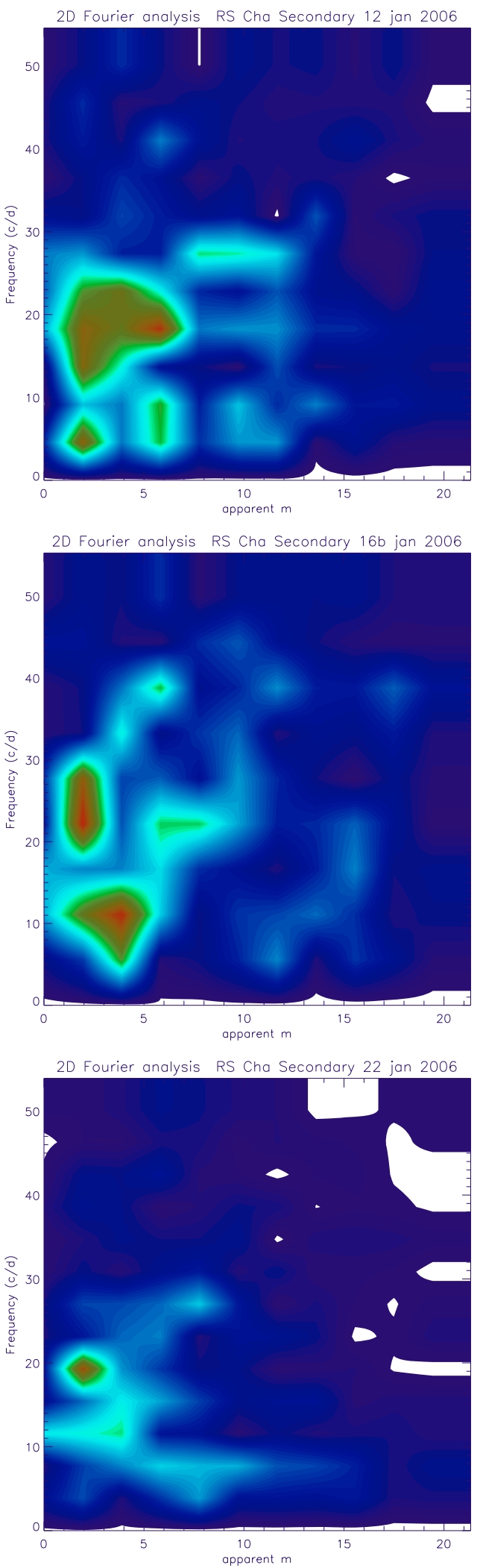

Fig. 14. F2D analysis on the secondary component of RS Cha for the nights of Jan. 12th, 16th and 22nd 2006.

\subsection{Mode identification with the FPF method}

We carried out a mode identification with the FPF method for the frequencies $f_{1}, f_{2 b}$, and $f_{3}$ and searched the same parameter space as for the primary component. The results are listed in

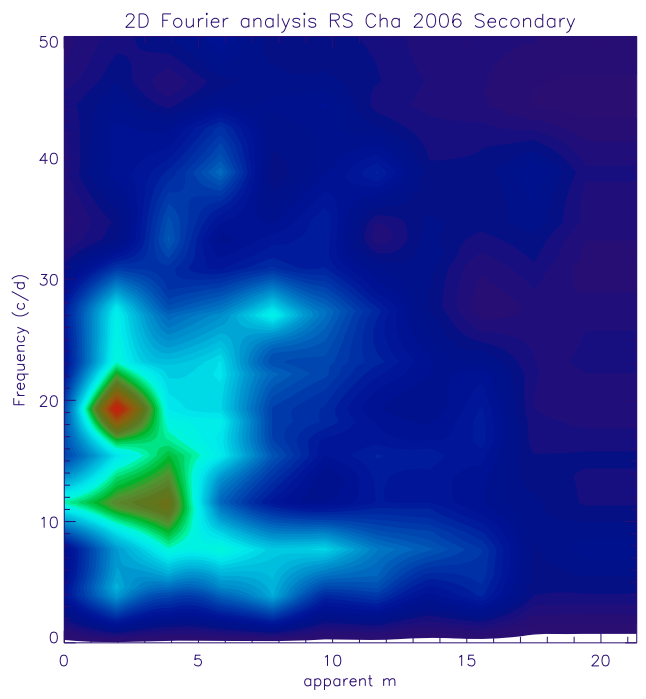

Fig. 15. Weighted F2D periodogram of the secondary component of RS Cha for the nights of Jan. 12th, 16th and 22nd 2006. White patches are only due to the applied cutoff values of the representation and have no further meaning.

Table 9. According to our mode identification, $f_{1}$ is a low-degree pro-grade mode, whereas the two other higher frequencies are high-degree pro-grade oscillation modes. In comparison to the mode identification of the primary, the values of the goodness of fit $\left(\chi_{v}^{2}\right)$ are higher for $f_{2 b}$ and $f_{3}$, which implies a lower reliability of the derived identification. For these two frequencies, the degree, $\ell$, is much better constrained than the value of $m$. Figure 13 shows the Fourier parameters of the observations and the two best fitting models for each mode.

\subsection{Results with the Fourier $2 D$ method}

Figure 14 shows the nightly F2D analysis for the nights of 12 th, 16th and 22nd of January 2006. The weighted F2D periodogram in Fig. 15 clearly exhibits the presence of 2 main frequencies around $12 \mathrm{~d}^{-1}$ and $19 \mathrm{~d}^{-1}$, which confirms the frequency determination by the FPF method for $f_{1}$ and $f_{2 b}$. The F2D method indicates an $\ell$ value of 2 or 3 for $f_{1}$ and confirms the FPF determination (within the error bars). However, the F2D method attributes an $\ell$ value of 0 to $f_{2 b}$ and identifies therefore this mode as radial, quite in contradiction with the FPF result for the same frequency, indicating a high degree $\ell=9-13$.

Table 10 summarises the results for the secondary component based on the F2D analysis.

\section{Discussion and conclusions}

We detected non-radial pulsations, for the first time, by direct spectroscopic means in both components of a binary Herbig Ae star. We identified profile variations in the primary and secondary components with a high level of confidence, two and three pulsation frequencies were found respectively. A first identification by two complementary methods revealed very different main pulsation modes for the two components: while the dominant mode of the primary component seems to be a high degree pro-grade mode with $\ell=10$ or 11 , the dominant mode of the secondary component is identified as a low degree mode with $\ell=0,1$ or 2 . Two different methods have been applied to perform a mode identification of both components. The Fourier 2D method as presented by Kennelly (1994) provides direct insights 
on the spatial structure of the oscillation. However, the derived apparent $|m|$ value seems to be linked to $\ell$ in the way described here-above, but more detailed simulations for different configurations have to be carried out in order to understand potential biases in the degree $\ell$ determination. We therefore privilege the $\ell$ determination as provided by the FPF method. Table 11 summarises the main results for the dominant pulsation modes of each component. As can be seen, both methods yield very different results on the determination of the degree $\ell$ of $f_{2 b}$ of the secondary component, perhaps due to an insensibility of the F2D method to tesseral mode signatures.

The precise redetermination of the binary orbit reveals a slight gradient change of the observed - calculated phase shift curve, which might be the signature of changes in the orbital period of the order of $\Delta P / P=(7.5 \pm 0.7) \times 10^{-6}$. This tendency, if confirmed, might be due to the presence of a third body in the RS Cha system or due to intrinsic orbital period changes, which then need to be explained.

Next steps require a precise redetermination of the fundamental parameters and a search for the intrinsic rotation periods of both components. The first frequencies are now known and their associated modes are constrained by the present work. In subsequent work, a numerical model will be developed in order to constrain the internal structure of both components.

Acknowledgements. We want to thank the University of Canterbury, New Zealand, for providing us access to their Mt. John $1 \mathrm{~m} \mathrm{McLellan} \mathrm{telescope,}$ but also to the staff of the Mt. John observatory for supporting us during the observing run. W.Z. is supported by the FP6 European Coordination Action HELAS and by the Research Council of the University of Leuven under grant GOA/2003/04

\section{References}

Alecian, E., Catala, C., Van't Veer-Menneret, C., Goupil, M.-J., \& Balona, L. 2005, A\&A, 442, 993

Alecian, E., Goupil, M.-J., Lebreton, Y., Dupret, M.-A., \& Catala, C. 2007a, A\&A, 465, 241

Alecian, E., Lebreton, Y., Goupil, M.-J., Dupret, M.-A., \& Catala, C. 2007b, A\&A, 473, 181

Alecian, E., Catala, C., Wade, G. A., et al. 2008, MNRAS, 385, 391

Andersen, J. 1975, A\&A, 44, 445

Böhm, T., \& Catala, C. 1995, A\&A, 301, 155

Böhm, T., \& Hirth, G. A. 1997, A\&A, 324, 177

Böhm, T., Catala, C., Carter, B., et al. 1996, A\&AS, 120, 431
Böhm, T., Catala, C., Balona, L., \& Carter, B. 2004, A\&A, 427, 907

Böhm, T., Dupret, M. A., \& Aynedjian, H. 2006, ed. A. R. Walker, \& G. Bono, Mem. S.A. It, 77, 362

Breger, M. 1972, ApJ, 171, 539

Breger, M., Stich, J., Garrido, R., Martin, B., et al. 1993, A\&A, 271, 482

Catala, C., Czarny, J., Felenbok, P., \& Praderie, F. 1986a, A\&A, 154, 103

Catala, C., Felenbok, P., Czarny, J., Talavera, A., \& Boesgaard, A. M. 1986b, ApJ, 308, 791

Catala, C. 2003, Ap\&SS, 284, 53

Catala, C., \& Kunasz, P. B. 1987, A\&A, 174, 158

Catala, C., Alecian, E., Donati, J.-F., et al. 2007, A\&A., 462, 293

Clausen, J. V., \& Nordstrom, B. 1980, A\&A, 83, 339

Donati, J.-F., Semel, M., Carter, B. D., Rees, D. E., \& Cameron, A. C. 1997, MNRAS, 291, 658

Finkenzeller, U., \& Mundt, R. 1984, A\&AS, 55, 109

Finkenzeller, U., \& Jankovics, I. 1984, A\&AS, 57, 285

Gilliland, R. L. 1986, ApJ, 300, 339

Gray, D. F. 2005, The Observation and Analysis of Stellar Photospheres, third edition (Cambridge University Press), 458

Herbig, G. H. 1960, ApJS, 4, 337

Horne, K. D. 1986, PASP, 98, 609

Iben, I. 1965, ApJ, 141, 993

Kennelly, E. J. 1994, Ph.D., University of British Columbia

Kennelly, E. J., Walker, G. A. H., Catala, C., et al. 2006, A\&A, 313, 571

Kurtz, D. W., \& Marang, F. 1995, MNRAS, 276, 191

Kurtz, D. W., \& Müller, M. 1999, MNRAS, 310, 1071

Kurtz, D. W., \& Catala, C. 2001, A\&A, 369, 981

Luhmann, K. L., \& Steeghts, D. 2004, ApJ, 609, 917

McInally, C. J., \& Austin, R. D. 1977, Information Bulletinon Variable Stars, 1334, 1

Marconi, M., \& Palla, F. 1998, A\&A, 507, L141

Marconi, M., Palla, F., \& Ripepi, V. 2002, Comm. Asteroseismology (Vienna), 141,13

Praderie, F., Talavera, A., Felenbok, P., Czarny, J., \& Boesgaard, A. M. 1982, ApJ, 254, 658

Ripepi, V., \& Marconi, M. 2003, Proc. 2nd Eddington workshop, Stellar Structure and Habitable Planet Finding, Palermo, ed. F. Favata, \& S. Aigrain, 397

Ribas, I., Jordi, C., Torra, J., \& Giménez, Á. 2000, MNRAS, 313, 99

Suran, M., Goupil, M., Baglin, A., Lebreton, Y., \& Catala, C. 2001, A\&A, 372, 233

Strom, S. E., Strom, K. M., Yost, J., Carrasco, L., \& Grasdalen, G. 1972, ApJ, 173,353

Wade, G. A., Drouin, D., Bagnulo, S., et al. 2005, A\&A, 442L, 31

Wade, G. A., Bagnulo, S., Drouin, D., Landstreet, J. D., \& Monin, D. 2007, MNRAS, 376, 1145

Zima, W. 2006, A\&A, 455, 235

Zima, W. 2008, Comm. Asteroseismology, 155

Zwintz, K. 2008, ApJ, 673, 1088

Zwintz, K., \& Weiss, W. 2003, Proc. 2nd Eddington workshop, Stellar Structure and Habitable Planet Finding, Palermo, ed. F. Favata, \& S. Aigrain, 105 